\title{
Interfacial Atomic Structure and Chemistry at Ceria Grain Boundaries
}

\author{
J.A. Aguiar, ${ }^{1,2}$ H. Yang* ${ }^{1}$ M.C. Sarahan, ${ }^{3}$ N.D. Browning ${ }^{1,2,4}$ \\ ${ }^{1}$ Dept. of Chemical Engr. and Mat. Sci., University of California - Davis, Davis, CA 95616 \\ ${ }^{2}$ Condensed Matter and Material Division, Lawrence Livermore National Laboratory, Livermore, \\ California 94550 \\ ${ }^{3}$ SuperSTEM Laboratory, STFC Daresbury, Keckwick Ln., Warrington, WA4 4AD, UK \\ ${ }^{4}$ Dept. of Molecular and Cellular Biology, University of California - Davis, Davis, CA 95616
}

Fluorite structured ceria $\left(\mathrm{CeO}_{2}\right)$ and ceria based compounds are strong candidates for both fuel cell, catalysis applications, and are considered surrogates for plutonia in next generation nuclear fuels. The ionic conductivity in nanocrystalline ceria is further influenced by impurity segregation [1] and grain size. [2-3]. Grain boundaries therefore have a predominant effect on many macroscopic electrical, mechanical, and chemical properties. Accurate determination of atomic grain boundary structure has a strong impact on understanding, extrapolating, and developing oxides for a variety of applications.

With the advent of aberration (Cs)-corrected STEM, experimental images with increasingly higher resolution, better signal-to-noise ratios, and contrast are now easily obtained in the latest transmission electron microscopes. HAADF-STEM images of nanocrystalline ceria shown in Fig.1 illustrates the atomic structure of [110] $90^{\circ}$ asymmetric grain boundaries. Using the structural unit model as suggested by Sutton et. al. [4-5] identified two principal types of structural units that are quasi-periodic along the grain boundary.

Although the repeating structural units have been clearly identified, each individual unit is not representative of the entire grain boundary structure due to the presence of structural variations. In order to analyze these structural variations, multivariate statistical analysis (MSA) has been identified as a technique as discussed in Sarahan et. al. to render the variations in the units as series of statistically decreasing principal component images. MSA has been used previously to analyze the Z-contrast images in the vicinity of dislocation cores in $\mathrm{SrTiO}_{3}$ [6]. Applying MSA to ceria we have identified a series of structural variations and have offered a depth structural interfacial analysis dissimilar to any other technique.

By applying MSA to the ceria grain boundaries, the resultant structural units are considered intrinsic structural variations in the formation of interfaces in fluorite oxides. Elucidating the validation of these proposed structural units we have coupled the use of atomic resolution electron energy loss (EEL) spectra with first principles and molecular dynamics calculations, as shown in Fig. 2, to understand and map the plausible ground state structures observed in fluorites. Based on the results of EELS we have further identified these grain boundaries as mobile entities and further have an effect on understanding the grain growth, migration, and the derived mechanical properties associated with cubic fluorites. The results of both MSA and the emerging coupled experimental and theoretical electronic structure analysis will be presented at the conference. 


\section{References:}

[1] Gerhardt, R. and A.S. Nowick, J. Am. Ceram. Soc. 69 (1986) 641.

[2] Chiang, Y.M.et. al. , Nanostruct. Mater. 9 (1997) 633.

[3] Bellino, M.G. et. al., Adv. Funct. Mater. 16 (2006) 107.

[4] Sutton, A.P. and V. Vitek, Philosophical Transactions of the Royal Society of London Series aMathematical Physical and Engineering Sciences. 309 (1983) 1.

[5] Browning, N.D. and S.J. Pennycook, J. Phys. D-Appl. Phys. 29 (1996) 1779.

[6] Sarahan, M.C., et al., Ultramicroscopy (2010)

[7] The authors acknowledge support of the National Center for Electron Microscopy, Lawrence Berkeley Lab, which is supported by the U.S. Department of Energy under Contract \# DE-AC0205CH11231. This work was performed under the auspices of the U.S. Department of Energy by Lawrence Livermore National Laboratory under Contract DE-AC52-07NA27344. This work is supported by the US DOE grant number DE-FG02-03ER46057.

Figure 1: HAADF STEM Image

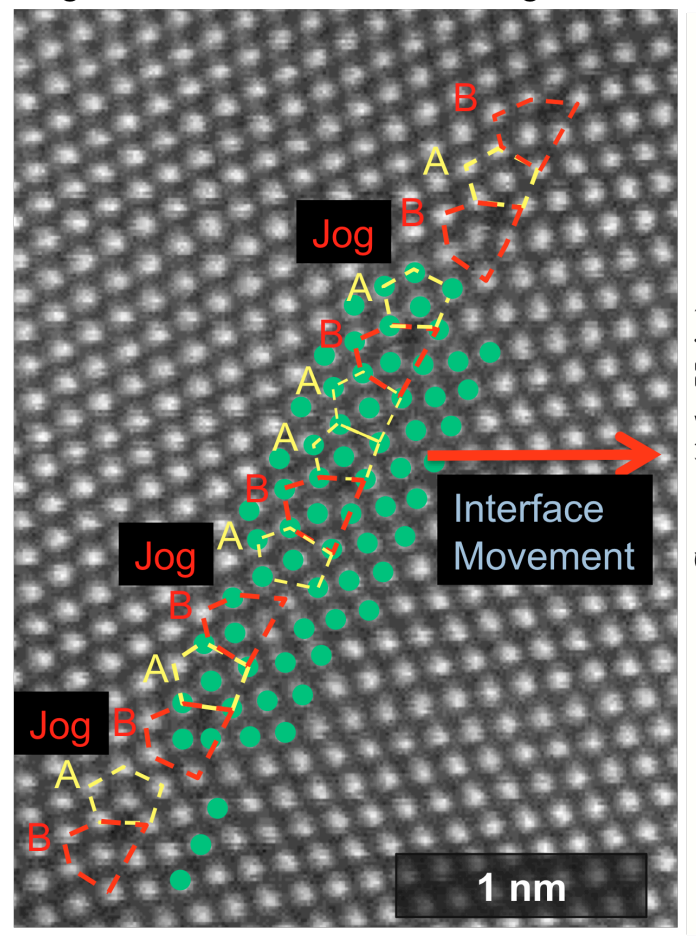

Figure 2: Oxygen K-Edge EEL Profiles

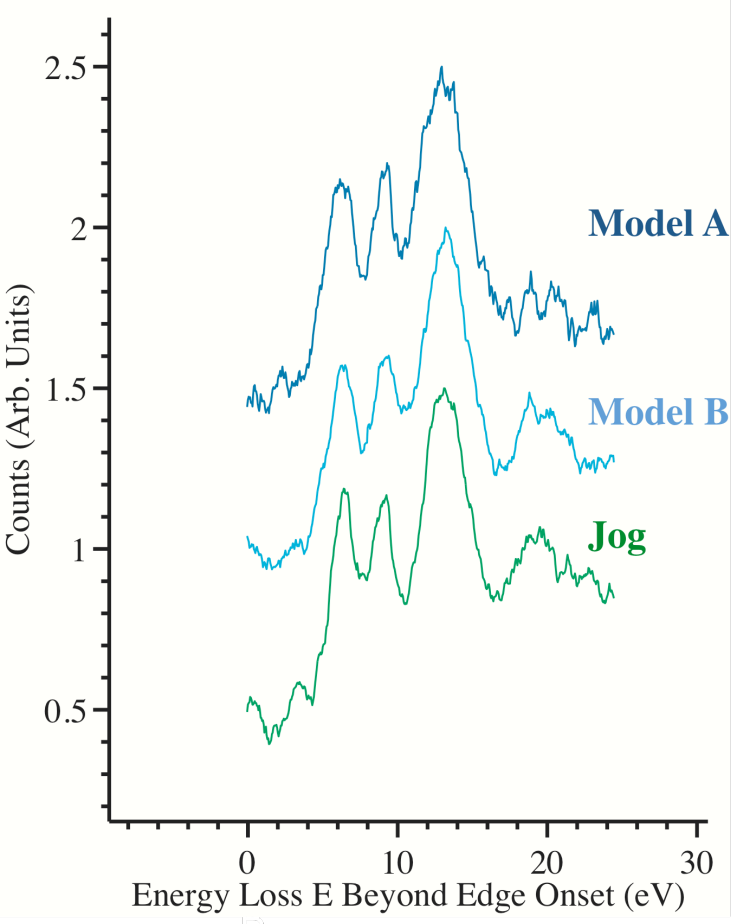

Figure 1: HAADF-STEM image of nanocrystalline $\mathrm{CeO}_{2}$, with the [110] $90^{\circ}$ assymmetric grain boundary resolved with a characteristic overlays of the identified principal structural units $\mathrm{A}$ and $\mathrm{B}$ in a quasiperiodic sequence.

Figure 2: Selected two dimensional EEL spectra taken from the vicinity of the grain boundary in the vicinity of the two suggested structure units and jogs, elucidating the fact that the grain boundary is mobile and shows characteristically different local binding environments. 